\title{
ADVERSE EVENTS ASSOCIATED WITH LABELED AND OFF-LABELED USE OF MEDICATIONS IN CHILDREN
}

\author{
M. Ramesh ${ }^{1,2}$, G. Nishitha ${ }^{1,2}$, D. Narayanappa ${ }^{3}$, G. Parthasarathi ${ }^{1,2}$ \\ ${ }^{1}$ Department of Pharmacy Practice, JSS University, JSS College of Pharmacy, ${ }^{2}$ Department of Clinical \\ Pharmacy, ${ }^{3}$ Department of Pediatrics, JSS Medical College Hospital, Mysore, India
}

Backgrounds and aims: The lack of specific drugs and labeling recommendations for the paediatric population is a long-standing problem and predisposes children to adverse effects. The study aimed to determine: the incidence, pattern, seriousness and predictors of adverse effects associated with labeled and off-labeled use of medication in children.

Methods: A prospective and intensive surveillance was conducted over nine months. Adverse event to labeled and unlicensed or off-label use of medications was detected and assessed for their incidence, seriousness and predictors of adverse events. Seriousness was asessed by using E2A guidelines of ICH-GCP.

Results: A total of 1504 medications administered to the 388 enrolled patients. Of these, $232(16 \%)$ medications were off-labeled use. Of the 82 adverse drug reactions (ADRs) reported from 58 patients, 53 $(64.6 \%)$ and $29(35.4 \%)$ were associated with labeled and off-labeled use of medications respectively. Incidence of ADR was found to be high with off-labeled use of medications (11.9\% vs $4.2 \%)$. Antibacterials [27 (33\%)] was the most commonly involved drug class. Skin and appendages [32 (31\%)] was found to be the most commonly affected organ system. Thirty three (40.2\%) ADRs were serious in nature. Patients aged 11-15 years [OR: $3.42(0.67-17.3) ; p<0.16)$, and administration of $\geq 4$ medications [OR: $1.17(0.52-2.5) ;(p<$ $0.69)$ were the predictors of ADRs in children.

Conclusions: Incidence of adverse events was high for off-labeled use of medication. Patients aged 11- 15 years and receiving $\geq 4$ medications were the predictors of ADRs in children. 